\title{
Evaluation of stable tungsten isotopes in the resolved resonance region
}

\author{
F. Emiliani ${ }^{1}$, K. Guber ${ }^{2}$, S. Kopecky ${ }^{1}$, C. Lampoudis ${ }^{1}$, C. Massimi ${ }^{3}$, P. Schillebeeckx ${ }^{1}$, K. Volev ${ }^{1,4}$ \\ ${ }^{1}$ European Commission, Joint Research Centre, Institute for Reference Materials and Measurements \\ (IRMM), Retieseweg 111, B-2440 Geel, Belgium \\ ${ }^{2}$ Oak Ridge National Laboratory, Oak Ridge, USA, TN-37831 \\ ${ }^{3}$ Department of Physics, University of Bologna and sezione INFN of Bologna, Via Irnerio 46, \\ Bologna, 40126 Italy \\ ${ }^{4}$ Institute for Nuclear Research and Nuclear Energy (INRNE), Sofia, Bulgaria
}

\begin{abstract}
In the last decade benchmark experiments and simulations, together with newly obtained neutron cross section data, have pointed out deficiencies in evaluated data files of $\mathrm{W}$ isotopes. The role of $\mathrm{W}$ as a fundamental structural material in different nuclear applications fully justifies a new evaluation of ${ }^{182,183,184,186} \mathrm{~W}$ neutron resonance parameters. In this regard transmission and capture cross section measurements on natural and enriched tungsten samples were performed at the GELINA facility of the EC-JRC-IRMM. A resonance parameter file used as input in the resonance shape analysis was prepared based on the available literature and adjusted in first instance to transmission data.
\end{abstract}

\section{Introduction}

An accurate knowledge of neutron induced cross sections of tungsten is important in different fields, from nuclear astrophysics to neutron dosimetry, to fission and fusion technology. For instance there is a rising interest in $\mathrm{W}$ as structural material in plasma facing components of fusion power plants and fusion experimental devices. A fundamental aspect to be considered for $\mathrm{W}$ and its alloy materials in fusion applications is the burn-up under neutron irradiation, which may result in the accumulation of residual elements which are potentially detrimental to the structural properties of the original material; neutrons can also induce the production of lighter particles, like charged particles and gases, which can diffuse through the bulk material and increase the brittleness of the material itself. Despite the importance of neutron induced cross sections of $\mathrm{W}$, the status of the data is not yet completely satisfactory. Several benchmark experiments in the last decade have pointed out deficiencies and discrepancies in evaluated data files; a rather recent evaluation in the fast neutron range, performed by Capote et al. (2007), underlined that no significant improvement in quality seems to be possible without new measurements [2]. Also preliminary results obtained at the GELINA facility have highlighted problems in the resolved resonance region databases $[3,4]$.

Our new evaluation is based on a quite well tested procedure at GELINA, consisting basically in a combined analysis of experimental data available in literature together with a resonance shape analysis of transmission and capture data. A good set of resonance parameters for W, used as starting values for the fitting procedure, has been obtained by combining results of the transmission 
measurement of Camarda et al. [5] and the capture measurements of Macklin et al. [6]. From transmission measurements the parameter $g \Gamma_{n}$ (proportional to the neutron width) values are deduced, whereas from capture measurements the capture kernel $g \Gamma_{n} \Gamma_{\gamma} /\left(\Gamma_{n}+\Gamma_{\gamma}\right)$ can be obtained. From reference [6] we also considered the average radiation width for the four different isotopes. For the parity assignment of a resonance we applied the Bollinger-Thomas approach to the $g \Gamma_{n}$ values. In first approximation we assumed the same value of the scattering radius for all $\mathrm{W}$ isotopes. Negative resonances have been introduced to match the coherent scattering length $b_{c o h}[7]$ and the capture cross section at thermal point $\sigma_{\gamma}^{0}[8]$. Scattering radii and negative resonance parameters, together with their interpretation, are totally linked to the resonance parameter file and thermal data of the present evaluation. With the above described approach and by simply adjusting to transmission data on natural samples the resonance energies and in minor cases the $\Gamma_{n}$, we obtained improvements compared to current resonance parameters, as already pointed out in reference [4]. Resonance parameters were obtained from a resonance shape analysis in REFIT [9]; the code is based on the Reich-Moore approximation of the R-matrix nuclear reaction theory, with the possibility to account for many phenomena which can affect the measurements (e.g. Doppler broadening and resolution of the TOF spectrometer).

\section{Transmission and capture experiments}

Transmission and capture cross section measurements on natural and enriched $\mathrm{W}$ samples were carried out at the neutron time of flight facility GELINA (Geel Electron LINear Accelerator). The linac accelerates electrons up to $150 \mathrm{MeV}$, which are compressed in bunches with up to $120 \mathrm{~A}$ peak currents and $1 \mathrm{~ns}$ width. These high energetic electrons generate Bremsstrahlung in a U-target, with subsequent production of neutrons. The slow neutron component is enhanced by using a water moderator canned in beryllium. The samples were metal discs of natural as well as enriched $\mathrm{W}$ with thickness ranging from 0.22 to $15 \mathrm{~mm}$. All measurements were performed with the moderated neutron beam. Transmission measurements were performed at 50 and $25 \mathrm{~m}$ flight path stations at 800 and $50 \mathrm{~Hz}$ machine frequency. A permanent ${ }^{10} \mathrm{~B}$ was used to eliminate neutrons from the previous burst. The beam was collimated to a diameter of $45 \mathrm{~mm}$ at the sample position. ${ }^{6} \mathrm{Li}$ glass scintillators enriched at $95 \%$ in ${ }^{6} \mathrm{Li}$ were used as neutron detectors. Capture measurements were performed at $10 \mathrm{~m}$ in 50 and $800 \mathrm{~Hz}$ regime, and at 30 and $60 \mathrm{~m}$ at $800 \mathrm{~Hz} . \mathrm{C}_{6} \mathrm{D}_{6}$ detectors were used as $\gamma$ detectors. At the same time, measurements of the energy dependent neutron spectrum were carried out with a ${ }^{10} \mathrm{~B}$ Frisch-gridded ionisation chamber placed at about $80 \mathrm{~cm}$ from the capture detection system.

From transmission measurements the experimental transmission factor is obtained as the ratio of the events counted with the sample inside the beam $C_{i n}$ ' and the events recorded in absence of sample $C_{\text {out }}$ ', already dead time corrected and normalized for the intensity of the incident neutron beam, after correcting for their background contributions $B_{\text {in }}{ }^{\prime}$ and $B_{\text {out }}$ :

$$
\mathrm{T}_{\text {exp }}=\frac{\mathrm{C}_{\text {in }}^{\prime}-\mathrm{B}_{\text {in }}^{\prime}}{\mathrm{C}_{\text {out }}^{\prime}-\mathrm{B}_{\text {out }}^{\prime}}
$$

In condition of good transmission geometry the background $B(t)$ components are well known, and the background analytical expression:

$$
\mathrm{B}(\mathrm{t})=\mathrm{B}_{0}+\mathrm{B}_{\gamma}(\mathrm{t})+\mathrm{B}_{\mathrm{n}}(\mathrm{t})
$$

is determined with the black resonance technique. $B_{0}$ is a time independent ambient component; the time dependent component $B_{\gamma}(t)$ and $B_{n}(t)$, are respectively due to the $2.2 \mathrm{MeV} \gamma$-ray emitted after neutron capture by hydrogen in the moderator and to scattered neutrons inside the detector station or coming from neighbouring flight paths. 
From capture measurements the experimental yield $Y_{\text {exp }}$ is deduced from the ratio of the observed response of the capture detectors and the neutron flux detector:

$$
\mathrm{Y}_{\exp }=\frac{\mathrm{N}_{\mathrm{c}}}{\varepsilon} \frac{\mathrm{C}_{\mathrm{W}}-\mathrm{B}_{\mathrm{W}}}{\mathrm{C}_{\varphi}-\mathrm{B}_{\varphi}} \mathrm{F}_{\varphi} \mathrm{Y}_{\varphi}
$$

In equation $3 C_{w}$ is the dead time corrected weighted response and $B_{w}$ is its background contribution; $C_{\varphi}$ is the dead time corrected neutron TOF spectrum and $B_{\varphi}$ the corresponding background. $F_{\varphi}$ accounts for the attenuation in the exit window of the ${ }^{10} \mathrm{~B}$ chamber and in the air between the chamber and the target; $\varepsilon$ is the capture detection efficiency; $Y_{\varphi}=\left[1-\exp \left(-n_{\varphi}\right.\right.$ $\left.\left.\sigma_{\text {tot }}\right)\right] \sigma_{\alpha} / \sigma_{\text {tot }}$ is the theoretical yield of the neutron flux detector $\left(n_{\varphi}\right.$ is the areal density of the ${ }^{10} \mathrm{~B}$ layer in the chamber, $\sigma_{\alpha}$ the cross section for the ${ }^{10} \mathrm{~B}(\mathrm{n}, \alpha)$ reaction and $\sigma_{\text {tot }}$ is the total cross section for neutron induced reactions in ${ }^{10} \mathrm{~B}$ ). There are basically three contributions to the background in capture measurements: a time independent component, due to environmental radiation and possible radioactivity in the sample and surroundings, which can be determined when the accelerator is not operating; a time dependent component which do not depend on the sample and it is estimated by sample-out measurements; a time and sample dependent component due to neutron and $\gamma$-rays which are detected after being scattered in the samples. In the considered range of energy, this component is mainly induced by scattered neutrons and increases with the neutron sensitivity of the detector. The smooth region, which is basically due to the potential scattering in the sample, is determined from measurements with a $0.5 \mathrm{~mm}$ thick ${ }^{208} \mathrm{~Pb}$ sample.

The data reduction for both transmission and capture data was carried out with the AGS code [10], a software package which can perform various operations on TOF-spectra as dead time correction, background subtraction and normalization, and which propagates the uncertainty at each step of the data reduction and provides the full covariance matrix.

\section{Results and discussion}

Preliminary observations show a rather good agreement between our initial resonance parameter file, - adjusted to transmission measurements on natural samples - and measurements in capture and transmission on enriched samples below $2.6 \mathrm{keV}$.

This agreement is in most of the cases better than the one showed by recent evaluated data files (an example is given in Fig.1). Resonance parameters from even tungsten isotopes need a minor adjustment.

In the evaluated data files isotope ${ }^{183} \mathrm{~W}$ seems to be the most problematic. In our starting parameter files, ${ }^{183} \mathrm{~W}$ resonances are in some cases clearly affected by a wrong spin assignment. In fact, in first instance we assigned the spin correspondent to the highest $g$ factor. A comparison between a thick and a thin sample can be used for spin assignment purposes. An example of a ${ }^{183} \mathrm{~W}$ resonance affected by wrong $J$ assignment can be seen in Fig.2. The shape of the resonance at $\sim$ $695.6 \mathrm{eV}$, which clearly belong to ${ }^{183} \mathrm{~W}$, is not well described by our initial choice of resonance parameters. A comparison two natural samples with different thickness $(0.2 \mathrm{~mm}$ and $0.4 \mathrm{~mm})$ lead us to the conclusion that the $\mathrm{J}$ assignment was wrong, where the initial choice was $\mathrm{J}=1$ for an $\mathrm{s}$ wave. 


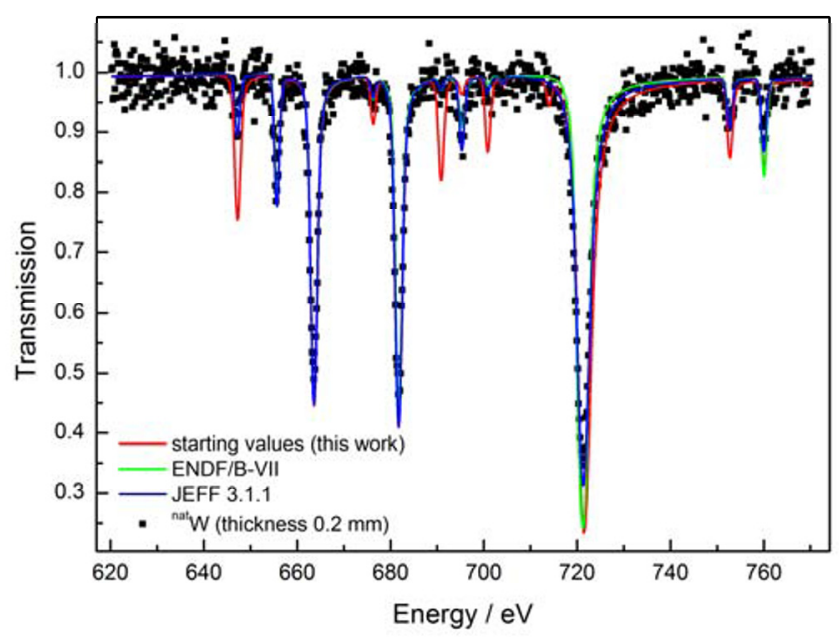

Fig.1 Transmission data obtained on a $0.2 \mathrm{~mm}$ thick ${ }^{\text {nat }} \mathrm{W}$ sample. The transmission measurement was performed at a $48 \mathrm{~m}$ flight path length with $800 \mathrm{~Hz}$ machine frequency. Our starting parameter file is compared with JEFF 3.1.1 and ENDF/B-VII.

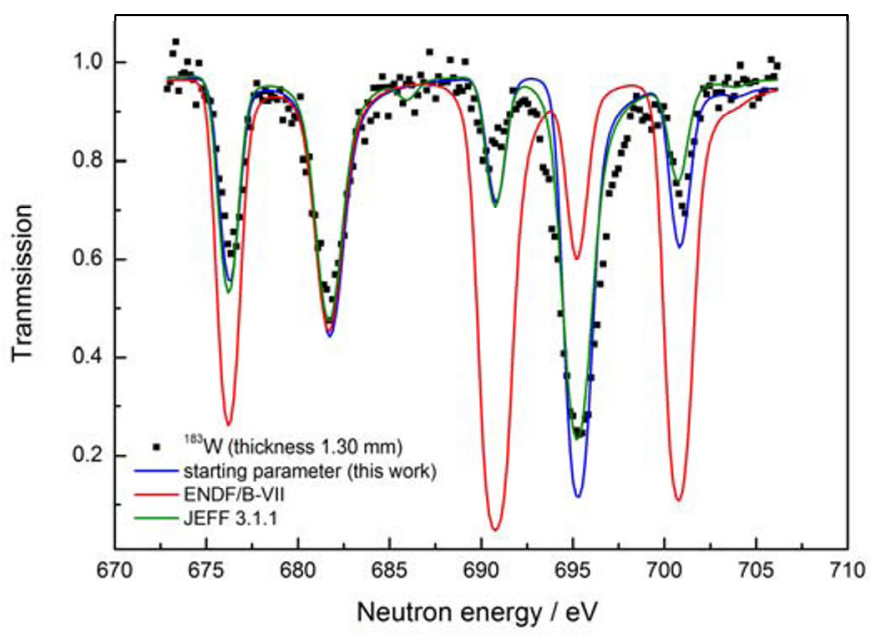

Fig.2 Transmission data obtained on a $1.3 \mathrm{~mm}$ thick ${ }^{183} \mathrm{~W}$ enriched sample. The transmission experiment was performed at a $48 \mathrm{~m}$ flight path length with $800 \mathrm{~Hz}$ machine frequency. The reproduction of the resonance shape with our initial parameter for the resonance at $\sim 695.6 \mathrm{eV}$ belonging to ${ }^{183} \mathrm{~W}$ is affected by a wrong $\mathrm{J}$ assignment $(\mathrm{J}=1)$.

By simply changing in $\mathrm{J}=0$ the total angular momentum of the neutron-nucleus system, and adjusting the $\Gamma_{\gamma}$ parameter to our capture data, it was possible to describe the resonance shape in transmission as well as in capture data (the case of capture is shown in Fig.3). 


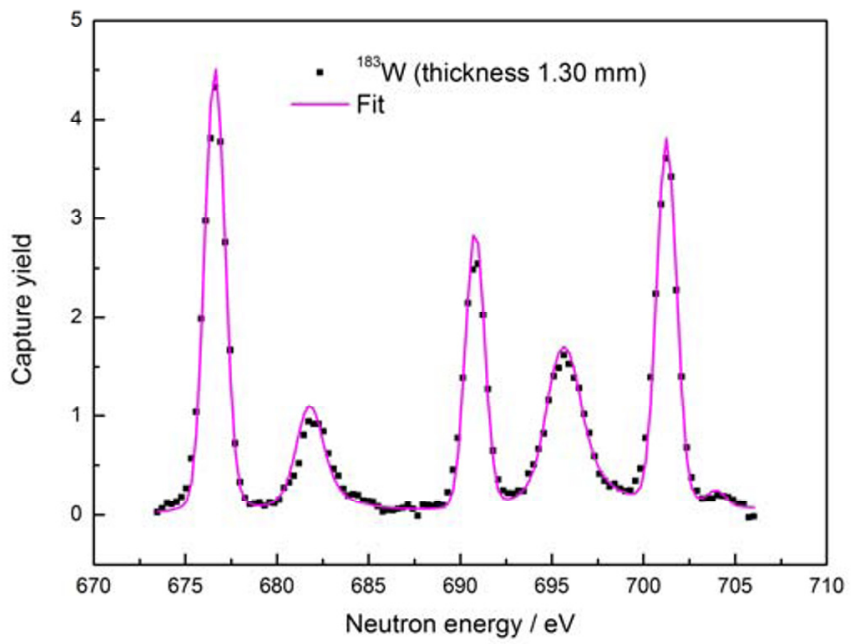

Fig.3 Capture yield obtained on a $1.3 \mathrm{~mm}$ thick ${ }^{183} \mathrm{~W}$ enriched sample. The capture measurement was performed at a $60 \mathrm{~m}$ flight path length with $800 \mathrm{~Hz}$ machine frequency. After changing the total angular momentum of the $\sim 695.6 \mathrm{eV}$ resonance of ${ }^{183} \mathrm{~W}$ into $\mathrm{J}=0$, it was possible to describe the capture data by slightly adjusting $\Gamma_{\gamma}$.

Another possible improvement to our parameter file will be the choice of the scattering radii obtained by optical model calculation, and the selection of suitable negative resonances to match the capture cross section at the thermal point.

\section{Acknowledgements}

This work was partly supported by the European Commission through the projects EUFRAT (FP7211499 and ERINDA (FP7-269499).

\section{References}

1. P. Batistoni, M. Angelone, L. Petrizzi and M. Pillon, J. Nucl. Mater., 329-333, 683-686 (2004).

2. R. Capote, A. Trkov, I. Kodeli, E. Soukhovitskii, L. C. Leal, M. Herman and D. W. Muir, Proceeding of the International Conference on Nuclear Data and Technology (2007), 690-692.

3. C. Lampoudis, K. Guber, S. Kopecky, P. Schillebeeckx and P. Siegler, J. Korean Phys. Soc., 59, 2, 1860-1863 (2011).

4. F. Emiliani, K. Guber, S. Kopecky, C. Lampoudis, P. Schillebeeckx, Proceeding of ICNC-2011, Edinburgh.

5. H. S. Camarda, H. I. Liou, G. Hacken, F. Rahn, W. Makofske, M. Slagowitz, S. Wynchank and J. Rainwater, Phys. Rev. C, 8, 1813-1826 (1973).

6. R. L. Macklin, D. M. Drake, E. D. Arthur, Nucl. Sci. Eng, 84, 98-119 (1983).

7. K. Knopf and W. Waschkowski, Zeit. Natur., 42a, 909-916 (1987).

8. S. F. Mughabghab, Atlas of Neutron Resonances, Elsevier, Amsterdam, The Netherlands (2006).

9. M. C. Moxon, T. C. Ware and C. J. Dean, "REFIT-2007" A Least-Square Fitting Program for Resonance Analysis of Neutron Transmission, Capture, Fission and Scattering Data.

10. B. Becker, C. Bastian, F. Emiliani, F. Gunsing, J. Heyse, K. Kauwenberghs, S. Kopecky, C. Lampoudis, C. Massimi, N. Otuka, P. Schillebeeckx and I. Sirako, submitted to JINST 